\title{
Research on Feature Extraction Curves and Surfaces in Reverse Engineering
}

\author{
Linzhe Ao \\ School of Mechatronics Engineering, Qiqihar University, \\ Qiqihar, China \\ 373803114@qq.com
}

\author{
Shigang Wang* \\ School of Mechatronics Engineering, Qiqihar University, \\ Qiqihar, China \\ hljwangsg@163.com
}

\begin{abstract}
Based on the measurement data reconstruction in reverse engineering has been widely used, the results contain a lot of noise data points are ubiquitous feature extraction as a key step in surface reconstruction more significance and difficulty. This paper describes the emergence and significance of reverse engineering, and illustrates the treatment stage surface reconstruction of key data processing steps and point cloud data. The importance of the feature extraction feature extraction method based on a detailed description and improvements.
\end{abstract}

Keywords-reverse engineering; surface reconstruction; data processing; point cloud data; feature extraction

\section{INTRODUCTION}

With the development of laser measurement technology and equipment, non-contact scanning product testing in the visualization, measurement applied more widely. It features high speed, the measured amount of data that can adequately represent parts of the surface information processing out vividly show the difference between the part and the design, the manufacturing precision visualization, and thus enhance and improve processing methods, processing play a guiding role, but also can improve efficiency and reduce costs. But usually the number of data points acquired by the above method is quite large and contain a certain measurement noise. In order to ensure the accuracy of the reconstruction model, usually using the appropriate measurement data prepossessing algorithm related.

\section{A. Reverse Engineering Technology Overview}

Reverse Engineering (Reverse Engineering, RE), also known as reverse engineering, reverse engineering, it refers to certain measurements or models of physical measurements, kind of CAD model reconstruction process by $3 \mathrm{D}$ geometric modeling method based on the measurement data, it is a product from the sample generation of digital information models and on the basis of product design and development and production of the entire process. Currently, it has become an important technology of digital

\author{
Shufeng Jiang \\ School of Mechatronics Engineering, Qiqihar University, \\ Qiqihar, China \\ jsf349@Sina.com \\ Shengyuan Jiang \\ School of Mechatronics Engineering, Qiqihar University, \\ Qiqihar, China \\ 446986768@qq.com
}

manufacturing and rapid response trend under 'CAD field and has developed into a relatively independent category. Here physical models can be mechanical products, the human body, animals and plants, artwork, terrain and so on. Most research on reverse engineering problems is concentrated in the geometry of the model that is the reconstruction of the actual products. In this sense, reverse engineering can be defined as: the physical into a generic term related to digital technology and the geometry of the CAD model reconstruction. Reverse engineering is compared to conventional CAD/CAM is concerned, the basic process is: First, the physical sample coordinate data measurement, obtained sample surface geometry data, and then put together on the measurement data, simplify, filter, triangle. and so presentment, since the measurement models are usually composed of a plurality of different types of surface composition, and thus the need for measurement data block, the data block basis points for surface fitting, final reconstructed model of the product. Fig. 1 is a flowchart of reverse engineering.

\section{B. Generate Reverse Engineering}

In the industrial era, the traditional product development process are the first by designers and marketing staff proposed product features and specifications based on market requirements, then all parts of the conceptual design, detailed design, prototype and trial to test design ideas and product performance and develop processing technology, complete the new product development process. The product development process known as forward engineering. However, for various reasons, there are still many products are not described by the CAD model, designers and manufacturers are often faced with the kind of sample. In order to adapt to the development of advanced manufacturing technology, through a certain way, the kind of sample into CAD model, then Lee CAD, CAM, RPM, CMIS advanced technology or management. Reverse engineering in this context came into being. 


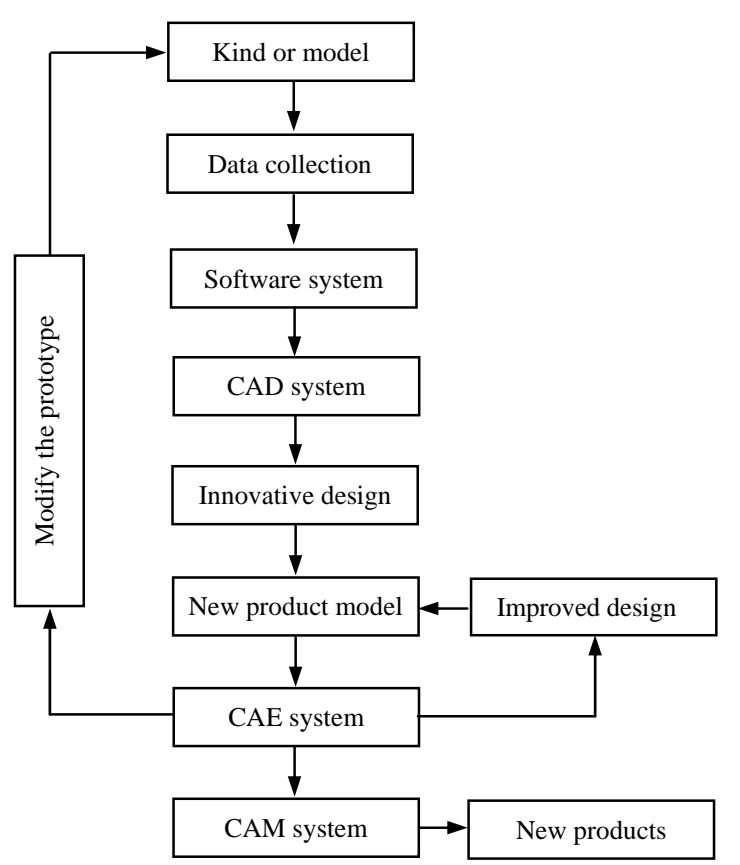

Figure 1. The entire process of reverse engineering

\section{Significance of Reverse Engineering}

Reverse kind of reverse engineering is the most important application areas, which means the general kind of reverse engineering reverse. Reverse kind can be defined as: In the absence of product original drawings, documents, or CAD model case, existing physical or sample measurement and analysis, counter-determined product geometry, process, physical and material properties, so as to Copy or develop similar new products in the process. Currently, the study focused on reverse engineering reverse geometry, that is how to accurately and efficiently capture complex threedimensional surface data from physical or samples, and then quickly get their CAD models. In this case, reverse engineering is the product of the physical sample or model geometry reverse process is the kind of digital technology into CAD models and geometry reconstruction in general. Reverse engineering the biggest difference is that traditional generic technologies: reverse engineering by restructuring parts of CAD product model, the prototype can be modified and re-designed to meet the design innovation, the purpose of product updates, imitation is the lowest level of reverse . Reverse engineering is the digestion and absorption of advanced technology for important technical means new product development, design and innovation. Reverse engineering in many industrial fields have a wide range of applications. For example, in only physical prototypes, no graphic document cases of sample finite element analysis, spare parts processing, mold manufacturing, or to modify the sample and analyze the sample assembly coordination with other parts of the modified between the like etc., we need to use reverse engineering process means the physical model into the model so as to improve the automation of design and manufacture. Rapid customization single product, the reverse engineering has been widely used, for example, the use of advanced measurement scanning device and model reconstruction software, you can quickly build human digital model, which can design and manufacture, such as helmets, clothing, etc. products, so that these products can meet the different needs of each client. Rapid mold manufacturing, toy design, art production, animal and plant simulation, medical diagnostics, human organs copy, geography topography and digital, film special effects, animation, and other areas of computer-aided detection, reverse engineering has been more and more applications.

In short, by means of reverse engineering the physical models into digital models, product design and manufacturing processes can make full use of the advantages of digital and intelligent adaptation, the process of information exchange requirements of integrated product set training and manufacturing. Reverse engineering of advanced data input device measuring equipment as a perproduct design, and CAD/CAM combine and form a closed loop product design and manufacturing systems, greatly improving the ability to respond quickly in product development.

\section{DATA PREPOSSESSING}

Shape of the surface that is obtained from the digitized kind enough to describe the object point data is a fundamental step in reverse engineering is a prerequisite for reconstruction of three-dimensional model. Depending on whether the contact surface of the real, three-dimensional surface data collection methods can be divided into contact and non-contact data acquisition data acquisition into two categories, based on contact mechanics have triggered deformation theory and continuous scanning data acquisition and field-based, ultrasonic waves data acquisition. Instead contact the main laser triangulation, laser ranging method, optical interferon, structured optics, image analysis method.

Typically, the three-dimensional coordinate measuring point set obtained three-dimensional space is called a point cloud (Point cloud). The minimum point cloud contains only one point, high-density point clouds up to several million data points. In order to effectively deal with various forms of point cloud, according to distribution of point cloud (such as arrangement, density, etc.) of the point cloud is divided into:

(a) Scattered point cloud, which is characterized by no clear distribution of geometry, was scattered disorder.

(b) Point Cloud, by a group of scanning lines, all of the scanning point scanning lines in the plane.

(c) Grid point cloud, point cloud all points of a uniform grid vertical correspond to the parameter field.

(d) Polygon point cloud, measuring points are distributed in a series of parallel planes, with a small segment of the same plane.

\section{FEATURE EXTRACTION OF MEANING}

Underlying extracting features simple geometric elements extracted directly from the measured data points, lines, surfaces, is the basic geometric units' level combination of features. High Composite feature refers to the sectional shape of the feature extraction by the 
underlying feature or combination of features vowels (cell), or will be directly used for parametric modeling curves, surfaces. B-rep unit/vowels characterized by CAD systems with specific engineering significance of the feature, which is characterized by the formation of high-level rules in accordance with a particular feature (stretching the rules, the rotation rule, sweeping rule, transition rules and composition rules, etc.) design made, or high-rise features a rep unit to $\mathrm{B}$ /vowels simple mapping feature space. It can be said, the underlying characteristics of reverse engineering CAD modeling features, then exploded high level, is characterized by a combination of high-level or low-level features of the complex, while a rep unit B/vowels characteristic feature is a positive level in accordance with the rules of the particular characteristics of the generated form design features to. Therefore, the reverse engineering feature is layered, corresponding to different levels have different characteristics space, the inter linkages between them, and there is a mapping between the different levels of feature space.

In reverse engineering, model reconstruction can be understood as a process: the object model based on reverse point cloud, rebuilding their geometric and topological information, and the process of reproduction characteristics. Characterized mainly refers to have a critical impact on surface modeling some local surface or curve. Transitional surface where it is customary to secondary surfaces and between surfaces collectively referred to as surface features, and the boundary lines of intersection between local surface and local surface is called the characteristic curve. Wherein the characteristic curve is based on the data block, the surface feature is the basis of modeling. These features of the reconstructed model quality play an important role. Feature extraction is mainly extracted from these characteristic curves and characteristic surfaces from the measured data points. Measurement data is filtered, and streamlined, the next step is to extract line features. Line features including the boundary lines of intersection between local surface and partial surface.

The most commonly used reverse engineering CAD modeling software can not accurately reproduce the characteristics of a surface model or additional local features, the lack of effective operational characteristics. In NURBS surface reconstruction represented by the same lack of recognition of the characteristics of the surface, even on the surface model can make more appropriate block is unified with free-form surfaces constructed for each block, shape retention and influence reconstruction model differential properties, increasing the cost of reconstruction. Therefore, in the reverse engineering of CAD surfaces (especially complex surface) reconstruction modeling, in order to achieve the desired results, it is necessary to reverse the object of extracting feature to feature-based modeling. How accurately reproduce features and additional design features is the field of reverse engineering to rebuild the core technology of surface hot research issues and applications.

\section{A. Feature Point Extraction Method}

Feature line extraction algorithm is based on triangular meshes using continuous curvature-based approach to achieve. The feature point extraction in two steps: First, if the angle between adjacent triangles triangular vector method illustrates one side is greater than a value, then the two vertical mark the edge of the feature point: Next, calculate each vertex of a triangle mesh normal vector, second order curvature vector and adjacent point estimate of the characteristic curve, calculate the surface curvature in the main and the main direction of the vertical, in the main direction of the main point is the curvature extremes feature points extracted feature points merge, connection to the initial characteristic curve.

\section{B. Calculation Feature Point Extraction}

In the triangular mesh model, there are some obvious features lines intersecting faces, and in the same direction with the characteristic triangle side lines. In this case, the angle between adjacent triangles features than the flat line at the edge of the angle between adjacent triangles smaller and triangle normal angle supplementary angle to each other. Therefore, we designed a user interaction required threshold $e$, when the normal vector angle is greater than $k$, the edge of the two vertical can be determined as a feature point (show in Fig. 2). When an edge is the boundary, the vector angle which method can be set to $180^{\circ}$. On each side of the triangle mesh to calculate the vector angle method can determine the initial part of the feature points and border points.

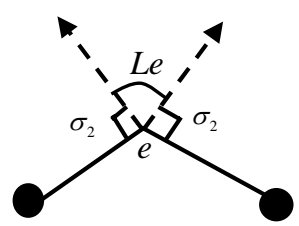

Figure 2. The angle between the two sides

Vector calculation method is simple, fast, and able to easily determine the boundary point, some simple model based on the extracted feature point normal can meet the requirements without the need for time-consuming calculation of curvature. However, it has a smooth transition or edge of the triangle inconsistent orientation characteristic lines can not handle the situation; we need to use the following curvature extremes and the second feature point extraction.

\section{Secondary Extraction}

Arbitrarily complex surface at a vertex at the local shape can be approximately described by a quadratic surface $S(u, v)=(u . v . h(u . v))$, Where $h(u . v)=a u^{2}+b u y+c v^{2}$, if a coefficient terms are zero, and then the secondary surface is a quadratic parabola. Since the square triangular mesh in the local coordinate system phuv a vertex at the h-axis is always vertex normal vector direction, and therefore need to estimate the surface normal at each vertex of direction in 
order to establish the point local coordinate system. To obtain surface at $P$ darboux structure, we must first estimate for each vertex of the triangle sheet $P_{1}$ normal.

Weighted overlay unit normal vector from the mechanical point of view given the formula Ke Yinglin, eliminating the situation when the side adjacent vertex dense sparse on the other side, the normal vector direction of deviation occur:

$$
N_{i}=\sum_{j=1}^{m} \frac{d_{j, j+1}}{d_{i, j}+d_{i, j+1}+d_{j, j+1}} \cdot n_{j}
$$

Has a unit force $n_{j}$ the same direction inside the triangle, the three vertical of the three component anti-parallel to the weight factor for component size $n_{j}$ ago, the triangle is in equilibrium state. This decomposition approach $n_{j}$ makes the transition vertex normal more evenly. This method of calculation of the mesh vertical normal draws mechanical balance thought: Suppose there is a unit normal vector $n_{j}$ with units to force the heart of the triangle, the three vertical of the three component anti parallel with $n_{j}$ especially bad for the normal vector calculated will $n_{j}$ coefficients weighting factor as component size, the triangle is in equilibrium state $n_{j}$ such a formula so that the resolving power transition vertex normal uniform, can reduce irregular elongated triangle affected.

In many documents, we have adopted an adjacent triangle normal and two gusset area weighted method to calculate the entry point normal vector, $N F_{j}$ is set $f_{j}$ triangle normal, $A_{j}$ is a triangular piece of $f$ the area, the vertex normal vector $N_{j} V_{j}$ is:

$$
N_{j}=\sum_{j=1}^{m} A_{j} \cdot N F_{j}
$$

After the experimental comparison, the curvature estimation algorithm in this paper is calculated using the formula mesh vertex normal for subsequent secondary surface fitting, resulting curvature value estimate is more accurate. In the estimation of the mesh surface normal vector $N_{j}$ after at the apex, you can create a mesh vertical $V_{j}$ local coordinate system phuv. Vertex $V_{j}$ in the local coordinate system of the coordinate values $\left(u_{j}, v_{j}, h_{j}\right)$. Then $u_{j}$ local conifer can obtain a least squares fit of the local coordinate neighborhood point through all adjacent points $(j=1,2 \ldots m)$ in mesh vertex $V_{j}$, since the distance nearer neighbors point to reflect the more local surface shape at the apex, so our algorithm to calculate the entry point normal when given the distance vertex $V_{j}$ and its neighbor to neighbor point between clever point $A_{h}$ corresponding weight w, so that you can get the sense of quadratic weighted least squares approximation surface. The weighted least squares error function $V_{j}$ and local conifer flail can be expressed as follows:

$$
o=\sum_{j=1}^{m}\left(\begin{array}{c}
u_{j} \\
v_{j} \\
a u_{j}^{2}+b u_{j}+v_{j}+c v_{j}^{2}+d u+e v
\end{array}\right)-\left.\left(\begin{array}{c}
u_{j} \\
v_{j} \\
h_{j}
\end{array}\right)\right|^{2} \cdot w_{j}(3)
$$

Among them: $w_{j}=e^{-d_{j} / \max \left(l_{j}\right)},(1 \leq j \leq m)$. So respectively the formula for variable partial derivative, take $\frac{\partial o}{\partial a}, \frac{\partial o}{\partial b}, \frac{\partial o}{\partial c}, \frac{\partial o}{\partial d}, \frac{\partial o}{\partial e}$ to give the corresponding linear equations, Gaussian elimination method for solving linear equations, you can get local quadratic surface $S(u, v)$ of the parameters $a, b, c, d$, and $e$.

After the establishment of the local surface quadraphonic surface $S(u, v)=(u, v, h(u, v))$ at the mesh vertices $P$, can determine the Darboux structure at that point $D(P)=\left(P, m_{1}\right.$, $\left.m_{2}, N, k_{1}, k_{2}\right)$. The structure can be judged by the Darboux the principal curvature extremes, as shown in Fig. $3, P_{i}$ point at the intersection $m_{1}$ direction and reverse the extension line of the triangle as $A, B$ (the actual space does not intersect the vertical projection of the intersection of the extension line and triangle triangle surface), and when the point $P_{i}$ principal curvatures $k_{1}$ the absolute value greater than $a, B$ two points on the absolute value of $m_{1}$ direction $k$ value $P_{i}$ at this point is in the direction of the curvature of the extreme points $m_{1}$, marked as $a$ feature point, $k$ value of the $a, B$ point by $V_{j}, V_{j}+k_{1}$ value $m_{1}$ in the direction of the linear combination is obtained. The method is also applicable to $k_{2}$, $m_{2}$ can determine whether $P_{i}$ in the direction of the feature point.

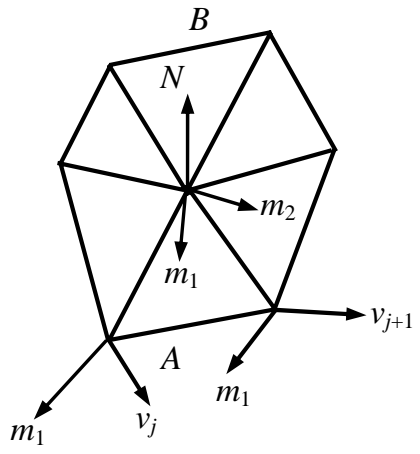

Figure 3. Calculation of principal curvature extremes

Because of the curvature calculation error, according to direct far more than actually required feature point of the extreme value judgments. If you join a local error elimination factor err, when comparing extremes, with (1$e r r) \times k_{1}$ instead $k_{1}$, with (1-err) $\times k_{2}$ instead of $k_{2}$, that is the main point of curvature extremes than $A, B$ points when the $k$ value to a certain extent, only that the point is a characteristic feature points can point err thinning dense areas, generally 0.05 to 0.1 is appropriate. To enhance the impact of large curvature region, to assign a weight to each extreme point of curvature value, equal to the weight of the absolute value of principal curvature extremes, if the principal curvatures are extremes in both directions, the absolute extreme value value sum. If you add the overall error threshold for each curvature extreme point, when a point right curvature value and maximum weight curvature value ratio is less than the threshold value, then it is removed from the feature point sequence, using the 
threshold can be removed curvature of the flat areas miscellaneous points (show in Fig. 4).

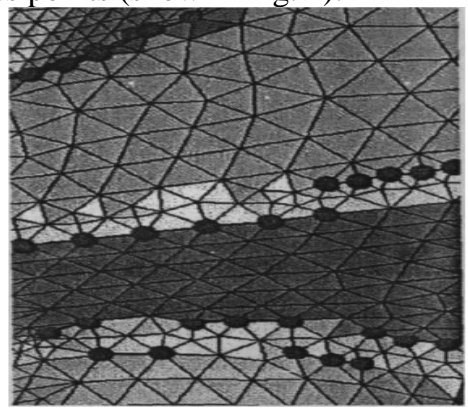

Figure 4. After two extracted feature points

\section{FEATURE EXTRACTION IN REVERSE MODELING EXAMPLES}

\section{A. Image Feature Extraction in Application Modeling a Flagon of Examples}

To carry out a flagon reverse design. The zuo.stl files and extracts data into contour together Imageware software. In Fig. 5, application of reverse engineering software for the flagon Image ware delete data scanning noise, move data from any coordinates to absolute coordinates, and the coordinates of the point cloud data alignment. After processing the coordinates and noise points, combined with UG flagon data modeling software.

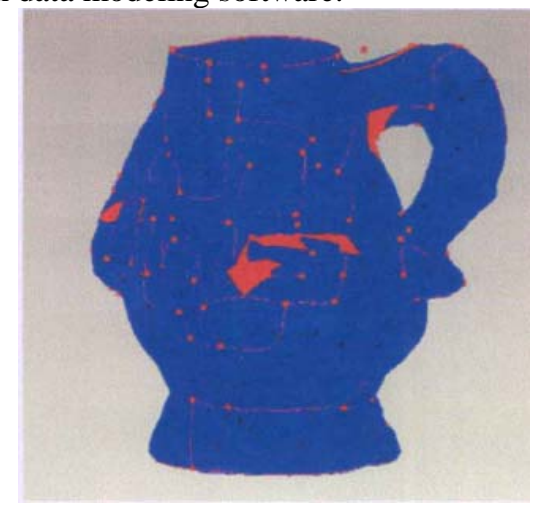

Figure 5. Flagon of point cloud data

\section{V.SUMMARY}

Reverse engineering technology has been widely used in the production practice, this paper research methods combining theory and practice, studied the reverse modeling process and feature surface, the first point clouds and presentment. Then the feature point extraction method and feature point extraction computing research, data calculated on the basis of the existing optimization resulting in a smoother feature points.

\section{ACKNOWLEDGMENT}

This work was supported by Project of Department of Education of Heilongjiang Province, China (Grant No.
12521597) and Qiqihar University youth teacher research startup project (Grant No. 2014k-M04). The Corresponding author is Shigang Wang, and the Corresponding email is hljwangsg@163.com.

\section{REFERENCES}

[1] Qing Chang, "Evergreen reverse engineering research and application of key technologies," Reverse Technology, vol. 15, 2010, pp. 35-36.

[2] Jinfeng Qian, Zhiyang Chen and Sanyuan Zhang etc, "Border point cloud data compression feature detection," China Image and Graphics, vol. 10, 2013, pp. 164-169.

[3] Xianying Zhang, Mingquan Zhou and Guohua Di etc, "Space boundary extraction method of triangular mesh," China Image and Graphics, vol. 8, 2003, pp. 1223-1226.

[4] Yixi Sui, "Reverse engineering characteristic curves and surfaces," Reverse Technology Research, vol. 10, 2012, pp. 112-113.

[5] Jiangxiong Li, "Reverse engineering of complex surface boundary line of automatic extraction," Mechanical Design and Manufacturing Engineering, vol. 29, 2000, pp. 26-28.

[6] Gang $\mathrm{Li}$, "Research on surface reconstruction based on reverse engineering," Shandong Technology, No. 16, 2009, pp. 57-58.

[7] Ping Yuan, "Research and application of reverse engineering," Kunming University, No. 13, 2002, pp. 23-24.

[8] Mingxiao Dong and Kangping Zhen, "A point of random noise filtering for point cloud data," China Image and Graphics, No. 2, 2004.

[9] Lei Wang and Yuan Xing, "Flatten reverse engineering data point cloud," Mold Technology, No. 1, 2004.

[10] Ying Cai, "CAD/CAM principles and applications," Machinery Industry Press, 1998. 